\title{
DC electric field distribution in planar dielectric in the presence of space charge
}

\author{
Z. Xu ${ }^{*}$, W. Choo and G. Chen \\ School of Electronics and Computer Science, University of Southampton, \\ Southampton, SO17 1BJ, United Kingdom \\ *E-mail : zx04r@ecs.soton.ac.uk
}

\begin{abstract}
Electric field in a dielectric material under dc conditions is determined by conductivity of the material. It is well known that the dc conductivity is a function of both temperature and electric field. This is generally not serious issue for a thinner planar sample as the temperature gradient is not great and the applied field is uniform across the sample. However, in the presence of space charge, electric field will be modified. As a result, the conductivity varies with the position, leading to change in electric field distribution. In this paper, we present a numerical simulation of electric field considering the effect of space charge. Space charge distributions were obtained from low density polyethylene (LDPE) and crosslinked polyethylene (XLPE) subjected to high dc voltage. Homocharge was formed in LDPE while heterocharge in XLPE. Electric field obtained from simulation has been compared with that without considering conductivity effect. It has been found that electric field retains its general shape. However, the maximum field has been significantly modified.
\end{abstract}

\section{INTRODUCTION}

Polymeric materials are gradually replacing traditional oil-impregnated paper insulation due to their excellent high dielectric strength and electrical resistivity in combination with some excellent physical properties such as resistance to cracking and moisture penetration [1]. However, good dielectric properties may bring some unwanted drawbacks to its performance under certain conditions. For example, the low charge carrier mobility and charge trapping within polymers give rise to space charge, resulting in localised electric stress enhancement. This is particularly true in the case where polymers are used under dc conditions such as dc power cable insulation; the formation of space charge may distort the electric field distribution across the cable insulation thickness. Such localised electric stress enhancement may lead to the premature failure of the cable insulation at stresses well below the anticipated or designed values [2].

For these reasons, space charge measurement has attracted the attention of researchers over the world in the past two decades and there is a large research base concentrated on the mechanism of space charge formation, migration and accumulation in film samples. It has generally been concluded that such space charge may result from electron (and hole) injection from the electrodes and electric field assisted ionization of the impurities in the material or from an inhomogeneous polarization [3-5].

At the present, majority of the fundamental researches on space charge have been carried out on planar samples due to their easy manufacture. The electric field in the presence of space charge has been calculated so far without considering conductivity variation due to space charge field. This will result in some error in electric field distribution as it is well known fact that electric field under dc conditions is determined by conductivity.

In the present paper, the influence of space charge field on the conductivity and subsequently electric field distribution in a planar sample has been considered. Space charge distributions in low density polyethylene (LDPE) and crosslinked polyethylene (XLPE) have been measured using the pulsed electroacoustic technique (PEA). A numerical simulation using COMSOL Multiphysics software package has been adopted to calculate the electric field distribution in the planar samples in the presence of space charge.

\section{GENERAL EXPRESSION FOR DC CONDUCTIVITY}

Under DC conditions the electric field is resistively graded. It is well known that dc conductivity is a strong function of both electric field and temperature [6]. The variation in conductivity with temperature of insulation has a major effect on field grading with load [7]. However, the field-dependence of the conductivity is relatively small and normally considered to be a second order effect.

Various models have been proposed to explain conductivity and its relationship with temperature and electric field. Conductivity given in equation (1) is well established in the electrical literature related to polymers and can be derived from a hopping theory model of conduction in the dielectric [8].

$$
\sigma(T, E)=A \exp \left(-\frac{\varphi q}{k T}\right) \frac{\sinh (B|E|)}{|E|}
$$

where $\mathrm{A}$ and $\mathrm{B}$ are constants, $\varphi$ the thermal activation energy in $\mathrm{eV}, \mathrm{q}$ the elementary charge, $\mathrm{T}$ the temperature in $\mathrm{K}, \mathrm{E}$ the electric field in $\mathrm{V} / \mathrm{m}$ and $\mathrm{k}$ the Bolzmann constant. 
This model is has been recently tested by Boggs et al [9]. It has been revealed that constants A, B and activation energy may change according to materials and their status. Based on experimental data, the parameters have been broadly classified into two groups; i.e. good dielectric and bad dielectric in [9].

\section{EXPERIMENTAL RESULTS}

Additive-free LDPE film of $90 \mu \mathrm{m}$ was purchased from GoodFellow and XLPE film of $135 \mu \mathrm{m}$ was peeled from a commercial $66 \mathrm{kV}$ XLPE cable. The XLPE cable did not go through degassing process therefore contains byproducts due to crosslinking. The samples were stressed at $10 \mathrm{kV}$ for a period of time. Space charge distributions were measured using the PEA technique [10]. The results are shown in Figures 1 and 2 respectively. In the case of LDPE sample, charge injection from the two electrodes takes place, leading to homocharge formation. The applied field is around $100 \mathrm{kV} / \mathrm{mm}$, significantly higher than the threshold field at which injection occurs. On the other hand, Figure 2 shows the dominated heterocharge formation in XLPE. After crosslinking process there will be formation of byproducts such as methane, cumylacohol, acetophenone etc. in the XLPE cable. Without degassing process these by-products may go ionisation process under a high electric field. Ionised species will move towards the opposite electrodes to form heterocharge. As indicated in LDPE, at $\sim 110 \mathrm{kV} / \mathrm{mm}$ field charge injection can also happen in XLPE, the observed charge distribution is the resultant effect of the two processes.

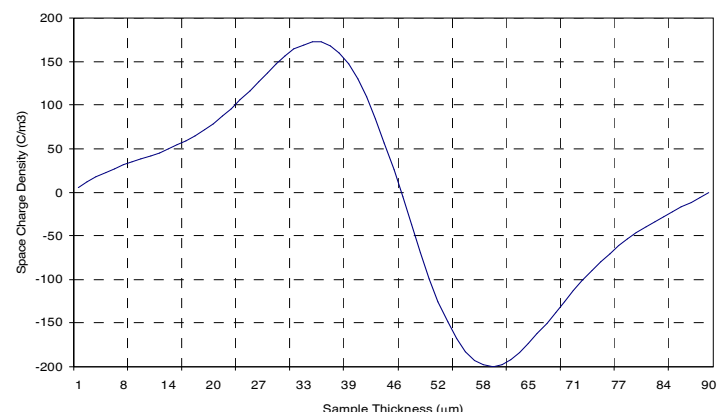

Figure 1: Space charge distribution in LDPE after the removal of an applied voltage of $10 \mathrm{kV}$ for 30 minutes (+10kV-LDPE-earth).

Once the space charge distribution, $\rho(x)$, is known in a material, the electric field, E(x), produced by space charge can be calculated based on Poisson's equation. For a planar sample Poisson's equation can be written as

$$
\frac{d E(x)}{d x}=\frac{\rho(x)}{\varepsilon_{0} \varepsilon_{r}}
$$

where $\varepsilon_{0}$ is the permittivity of vacuum and $\varepsilon_{\mathrm{r}}$ the relative permittivity of the material. Generally, it is believed that the field-dependence of the conductivity is relatively small, the total field in the published work is obtained by summing the applied field and this field due to space charge. Figure 3 shows the total electric field distribution for the above charge distributions. It can be seen that in the case of homocharge the maximum electric field occurs at the meeting point in the bulk of the LDPE sample. However, for heterocharge the maximum electric field occurs at the interface between anode and the XLPE due to large amount of negative charge accumulation in the region adjacent to the anode..

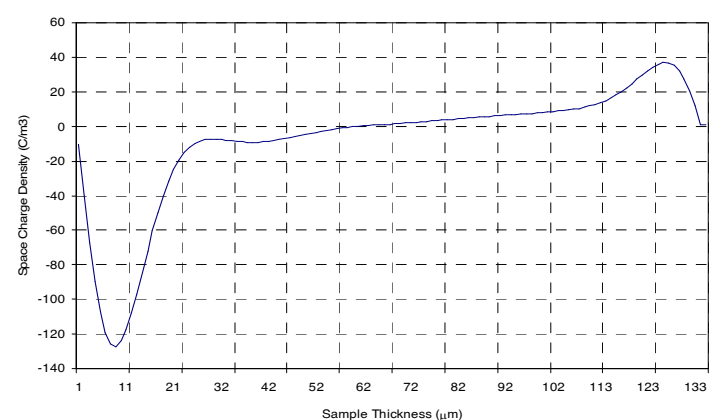

Figure 2: Space charge distribution in XLPE after the removal of an applied voltage of $10 \mathrm{kV}$ for 30 minutes (+10kV-XLPE-earth).

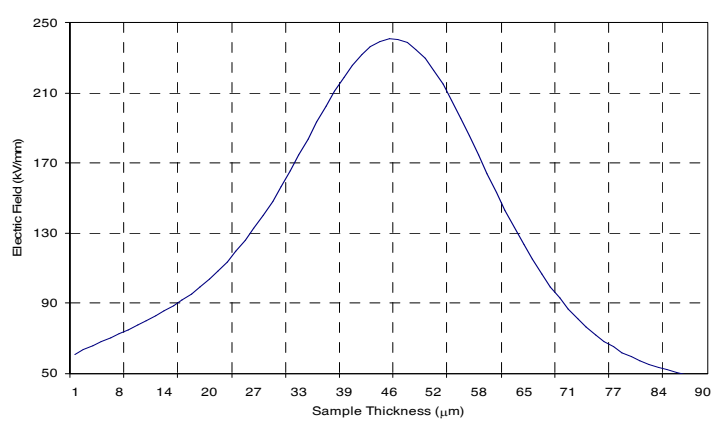

(a) LDPE

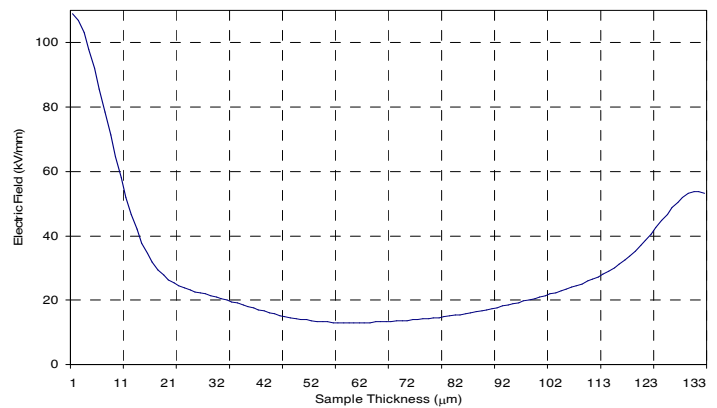

(b) XLPE

Figure 3: Electric field distributions in two samples.

\section{SIMULATION RESULTS AND DISCUSSION}

In the presence of space charge electric field in some regions shows a significant enhancement. The field 
effect on conductivity may be no longer small. However, this is not an easy task as the problem involves coupling. The electric field due to space charge affects the conductivity and the variation in conductivity will change electric field distribution. To accurately determine the electric field in the presence of space charge, COMSOL Multiphysics software package has been used. Three steps have been adopted. (i) Calculation of the electric field due to space charge based on Poisson's equation. (ii) The electric field obtained from (i) is incorporated into conductivity equation to determine the electric field arisen from the applied voltage. (iii) Finally adding the two fields to form the total electric field.

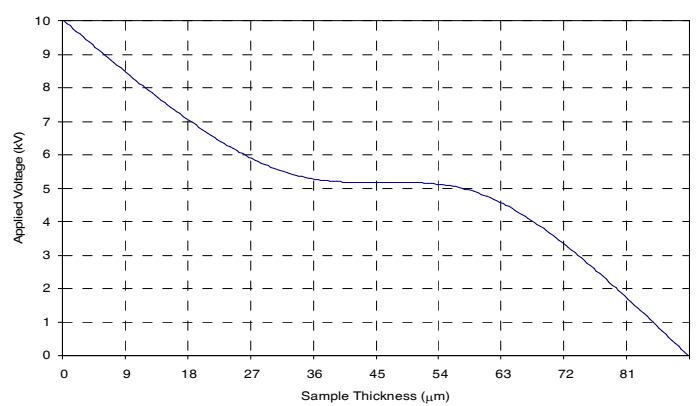

(a) Voltage distribution

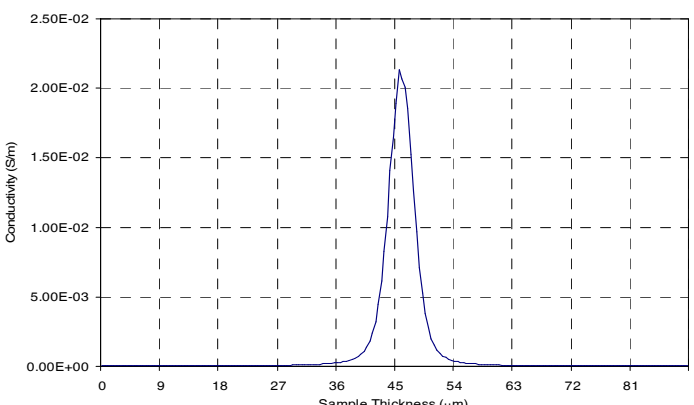

(b) Conductivity distribution

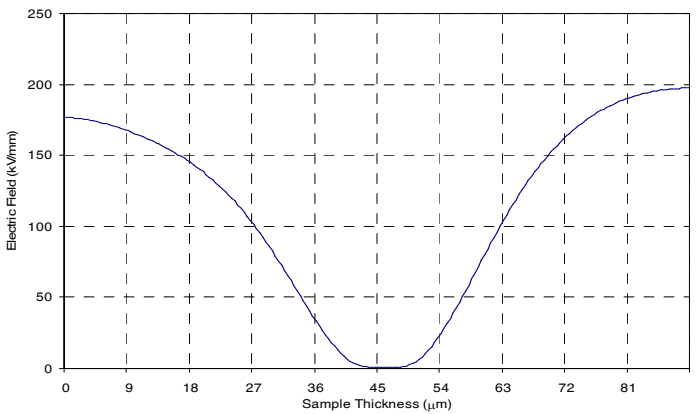

(c) Electric field distribution due to applied voltage

Figure 4: Simulation results for LDPE considering the field-dependent conductivity.

In the present simulation we have adopted parameters for conductivity expression from a good dielectric material [9]. Figure 4 shows the simulation results when $10 \mathrm{kV}$ is applied to LDPE sample. After considering the space charge field, the conductivity of the material is no longer uniform. The maximum conductivity occurs in the middle of the sample corresponding to the maximum electric field produced by space charge. Local conductivity can be as high as $2 \times 10^{-2} \mathrm{~S} / \mathrm{m}$. Consequently, the electric field due to the applied voltage is modified, showing the minimum in the middle of the sample. The voltage profile across the sample is severely deviated from the linear.

Figure 5 illustrates the total electric field distribution across the LDPE sample, together with the result calculated without considering the field-dependent conductivity. It is evident that after considering the field-dependent conductivity the high electric field region is suppressed while the lower field region is enhanced. Significant difference can be observed in the maximum field. The reduction in the maximum electric field after considering the field-dependent conductivity is as much as $46 \%$. The effect of the field-dependent conductivity is to make field distribution more uniform across the insulation.

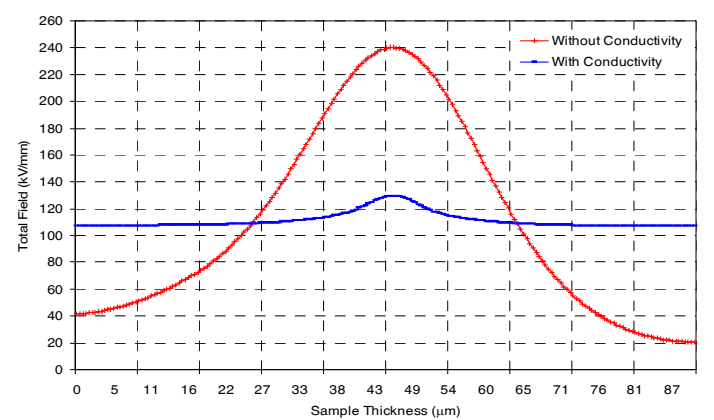

Figure 5: Total electric field in LDPE in the presence of space charge.

Figure 6 shows simulation results for XLPE based on the space charge distribution in Figure 2. In this case, the maximum conductivity occurs at the interface region close to the anode as shown in Figure 6 (b). The electric field due to the applied voltage shows the maximum in the middle of the XLPE sample and minimum at the interface where the maximum conductivity presents. The voltage profile shows slight nonlinear.

The total electric field from the simulation is given in Figure 7. For comparison reason the electric field without considering the field-dependent conductivity is also shown in Figure 7. The feature observed in the case of the presence of homocharge in LDPE remains true. The maximum electric field reduction is around 44\%, significantly less than that in LDPE sample.

The parameters used in the conductivity expression may have significant influence on the electric field distribution. The conductivity for a good dielectric has a stronger field dependence on electric field than a bad dielectric [9]. As the parameters used in the present simulation were taken from a good dielectric this may explain why the significant field modification has taken place for both LDPE and XLPE samples. 


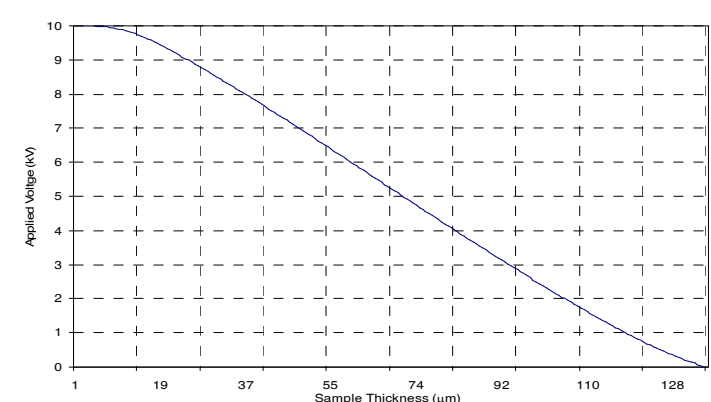

(a) Voltage distribution

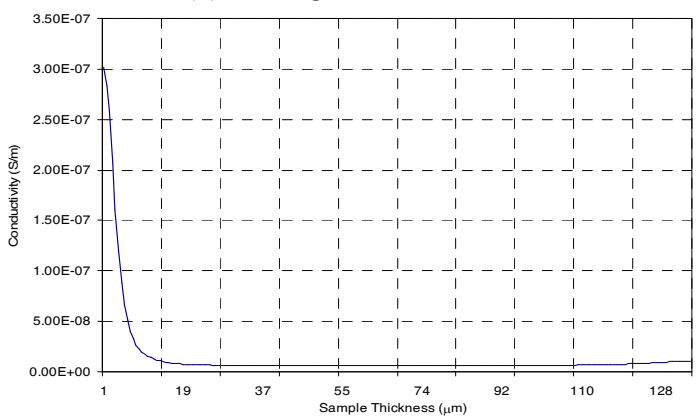

(b) Conductivity distribution

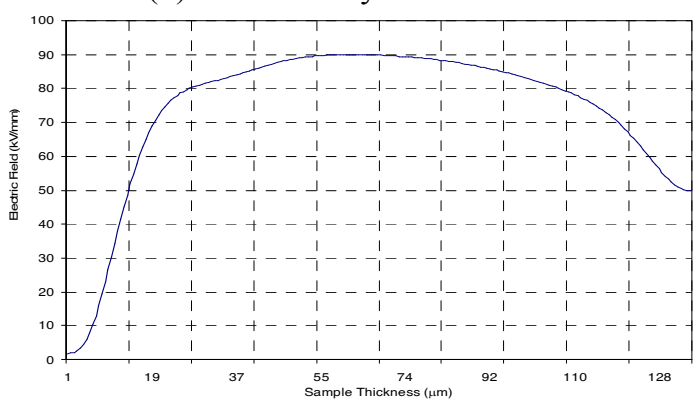

(c) Electric field distribution due to applied voltage

Figure 6: Simulation results for XLPE considering the field-dependent conductivity.

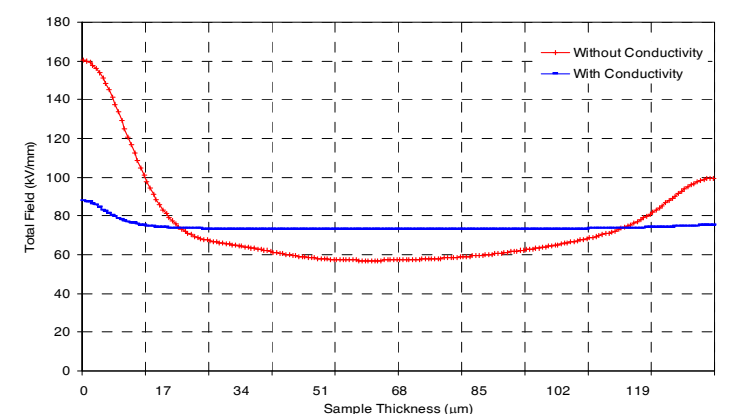

Figure 7: Total electric field distribution in XLPE in the presence of space charge.

In the present study, we have not considered the presence of a temperature gradient due to thinner samples investigated. The influence of a temperature gradient on the electric field distribution in the presence of space charge will be studied in future for thick samples such as polymeric power cables. More importantly, a significant field distortion may occur upon the polarity reversal.

\section{CONCLUSION}

We have measured space charge in LDPE and XLPE samples after at an applied voltage of $10 \mathrm{kV}$ for 30 minutes using the PEA technique. Homocharge has been formed in LDPE due to charge injection from the two electrodes while heterocharge formed in XLPE due to ionisation of crosslinking by-products. To obtain an accurate electric field distribution, the influence of space charge field on conductivity has been considered by simulation using COMSOL Multiphysics software package. Results reveal that the electric fields in both cases have been significantly modified. The fielddependent conductivity tends to reduce the maximum field and increase field magnitude in the lower field region. Overall it tends to smoothen the electric field distribution and the extent of modification depends on the magnitude of space charge field.

\section{REFERENCES}

[1] T. L. Hanley, R. P. Burford, R. J. Fleming and K. W. Barber, "A general review of polymeric insulation for use in HVDC cables", IEEE Electrical Insulation Magazine, Vol. 19, No. 1, pp. 13-24, 2003

[2] T. Tanaka and A. Greenwood, Advanced power cable technology, Vol. 1, CRC Press inc. USA, 1983.

[3] T. Mizutani, "Space charge measurement techniques and space charge in polyethylene", IEEE Trans. on DEI., Vol.1, pp.923-933, 1994.

[4] Y. Li and T. Takada, "Experimental observation of charge transport and injection in XLPE at polarity reversal", J. Phys. D: Appl. Phys. Vol.25, pp. 704-716, 1992.

[5] Y. Tanaka, Y. Li, T. Takada and M. Ikeda, "Space charge distribution in low-density polyethylene with charge-injection suppression layers", J. Phys. D: Appl. Phys., Vol.28, pp.12321238, 1995.

[6] B. M. Weedy and D. Chu, "HVDC extruded cables - parameters for determination of stresses", IEEE Trans PAS. Vol. 103, pp. 662667, 1984.

[7] B. M. Weedy, Underground Transmission of Electric Power. New. York: Wiley, 1980.

[8] A. R. Blythe and D. Bloor, Electrical Properties of Polymers, $2^{\text {nd }}$ Edition, Cambridge University Press, 2005.

[9] S. Boggs, D. Damon, J. Hjerrild, J. Holbol and M. Henriksen, "Effect of insulation properties on the field grading of solid dielectric DC cable.", IEEE TPD, Vol. 16, No.4, pp.456-461, 2001.

[10] G. Chen, Y. L. Chong and M. Fu, Calibration of the Pulsed Electroacoustic Technique in the Presence of Trapped Charge, Measurement Science and Technology, Vol. 17 pp.974-980, 2006. 